\title{
A PROPOS DE 10 CAS DE CANCER DU PENIS : REVUE DE LA LITTERATURE
}

\author{
J. Bellin* A. Elbikri** G. Arvis** \\ *Unité de Chirurgie Urologique Centre Hospitalier Marc Jacquet, MELUN \\ **Service d'Urologie - Hôpital St Antoine, rue du faubourg St Antoine, 75012 PARIS
}

\begin{abstract}
ABOUT 10 CASES OF PENILE CANCER : A LITTERATURE OVERVIEW. The epidermoid cancer of the male penis is a little frequent tumor. At early stage, the treatment is conservative (curi therapy and laser therapy). At advanced stage the treatment of the primary lesions is amputation. Prognosis depends on the presence or absence of lymph nodes metastasis. The management of lymph nodes metastatis is still controversial. Radical lymph nodes excision is the only effective treatment, but associated with high morbidity. The detection of papilloma gene virus in the tumor incites to search for such virus lesions by peniscopy and to treat them. Key words : epidermoid cancer, penis, node, therapy. Andrologie, 1991, 1 : 71-73.
\end{abstract}

Le cancer épidermoïde du pénis est peu fréquent (23). Son pronostic lié à l'envahissement ganglionnaire est redoutable car les patients consultent souvent tardivement.

L'attitude thérapeutique est encore controversée. Si l'amputation partielle ou totale du pénis avec ses conséquences psychologiques traite le problème local au stade avancée (3 et 4 de Jackson) quelques auteurs, au stade précoce, préconisent une curiethérapie, une radiothérapie ou une photocoagulation au laser $(2,9,16,21$. $26,27)$.

L'attitude vis à vis des ganglions est tout aussi controversée. Le drainage lymphatique s'effectue vers les chaines inguinales superficielies. Les ganglions satellites de la crosse de la saphène seraient les premiers relais envahis (4). L'envahissement ou la récidive ganglionnaire inguinale est péjorative. Elle expose à des complications lymphatiques locales, artérielles et veineuses (6). Certains auteurs comme Srinivas $(24,25)$ sont partisans du curage systématique ou de biopsies, mais d'autres, comme Périnetti (20) d'une simple surveillance devant l'existence de métastases ganglionnaires secondaires malgré un curage inguinal initialement négatif. Il nous a semblé intéressant de rapporter 10 cas de tumeur du pénis traités à l'Hôpital Saint Antoine, et de revoir la littérature à ce propos.

\section{SUJETS}

Depuis 10 ans, 12 patients ont été hospitalisés à l'Hôpital SAINT ANTOINE pour une tumeur du pénis, incluant 10 cancers épidermoïdes. Les 2 autres cas concernaient des localisations secondaires de tumeurs vésicales et n'ont pas été retenus. Ces 10 cas ne comprenaient pas de tumeurs superficielles ou de carcinomes in situ.

L'âge des patients variait de 37 à 87 ans avec une moyenne de 60 ans. Si les patients étaient pour la plupart âgés, un patient n'avait que 37 ans ef deux avaient moins de 50 ans. Le retard au diagnostic est surprenant : 7 patients ont consulté dans les 3 à 12 mois et 3 après plus d'un an. La présence d'une volumineuse tumeur, ou la surinfection d'un phimosis serré est le principal motif de consultation. Si notre série concerne surtout des sujets de plus de 70 ans et de condition sociale modeste, pouvant expliquer ce retard, les 3 patients jeunes ont aussi consulté tardivement par négligence ou complexe.

Dans 5 cas, la tumeur intéressait le gland avec déjà dans 4 cas extension aux corps caverneux, et dans 2 cas au sillon balano-préputial et dans 3 cas au prépuce.

La tumeur était 5 fois bourgeonnante ou ulcérante. Un seul patient avait été circonsis un an auparavant. Il s'agissait en fait d'une récidive tumorale après circoncision soulignant l'importance de l'examen histologique des prépuces infectés chez le sujet âgé.

\section{TRAITEMENTS}

Tous les cas ont été traités chirurgicalement : deux fois par amputation totale avec uréthrostomie périnéale,

sept fois par amputation partielle,

une fois par ablation du prépuce.

Une marge d'au moins $2 \mathrm{~cm}$ a toujours été respectée lors des résections.

5 carcinomes étaient différenciés, 4 peu différenciés, 1 indifférencié.

Tous les patients subirent d'emblée un curage ganglionnaire inguinal superficiel qui fût positif dạns $70 \%$ des cas. Sur les trois patients initialement indemnes d'envahissement ganglionnaire $(30 \%)$ deux développèrent secondairement des localisations ganglionnaires, un seul reste exempt de métastases et a survécu. Un patient reçu une chimiothérapie en pré et post-opératoire, un en post-opératoire. 8 furent traités par radiothérapie de complément.

\section{RESULTATS}

Si aucune mortalité post-opératoire n'a été notée, dans notre série de patients âgés consultant à un stade avancé, le pronostic fut sombre. Sur 7 patients $\mathrm{N}+$ d'emblée (stade III et IV de Jackson), deux décédèrent dans les six mois (un de son cancer, le second d'un cancer O.R.L. assacié). 5 survécurent plus de trois ans $(70 \%)$, 4 décédèrent de l'évolution de leur cancer et 1 de son âge avancé (accident vasculaire, pneumopathie et septicémie). Aucun des patient $\mathrm{N}+$ ne survécut plus de 5 ans.

Sur trois patients $\mathrm{N}-, 2$ décédèrent de complications liées à l'âge. Un seul patient survécut et est encore vivant 7 ans après son traitement, exempt de récidive ganglionnaire.

Les complications observées sont liées à l'âge des patients, aux récidives ganglionnaires et aux séquelles du traitement chirurgical.

Trois récidives ganglionnaires furent source de plaie atone et de nécrose inguinale. Pour un patient le risque de rupture de l'artère fémorale fit discuter un pontage extra-anatomique.

Un patient âgé et diabétique nécessita une reprise pour nécrose du moignon d'amputation.

Deux patients nécessitèrent une plastie du néo méat urétral sténosé.

Un patient développa un lymphocèle scrotal, deux patients une thrombophlébite.

Le retentissement sexuel pose de gros problèmes. Tous les patients jeunes ont cessé leur activité sexuelle et ont été pris en charge par le psychologue attaché à notre unité. Une composante dépressive a même dû être traitée chez deux de ces patients jeunes. Chez les sujets plus âgés, l'acceptation du geste chirurgical nécessita l'intervention du psychologue dans deux cas qui refusaient d'emblée l'acte chirurgical.

Le pronostic de ce cancer reste sombre, l'envahissement ganglionnaire, le stade, le degré d'infiltration de la tumeur, le degré de différentiation (11) et l'âge avancé des patients conditionnent le pronostic. 


\section{DISCUSSION}

Aspects cliniques et thérapeutiques : Les tumeurs épidermoïdes du pénis sont peu fréquentes : moins de $1 \%$ des cancers de l'adulte (23).

Leur traitement est controversé. Aux stades précoces (I et II de Jackson), l'amputation totale ou partielle assure $90 \%$ à $95 \%$ de guérison lorsque la résection passe à plus de $2 \mathrm{~cm}$ de la tumeur et lorsqu'il n'existe pas d'envahissement ganglionnaire $(4,5)$. La précocité du traitement conditionne donc, comme dans tous les cancers, le pronostic.

Le retentissement psychologique, surtout chez les sujets jeunes qui consultent pour de petites tumeurs du gland ou du sillon balano-préputial, a conduit à développer des techniques conservatrices telles que la curiethérapie à l'iridium développée par Pierquin et préconisée par El Demiry $(10,21)$. Elle assure $89 \%$ de survie à 5 ans lorsque la tumeur est localisée au gland mais expose aux récidives ( $20 \%$ ), et aux séquelles post-radiques. La photocoagulation au laser Yag avec petites tumeurs du gland (récidive dans $30 \%$ des cas) a été utilisée par Staehler (26) chez six patients avec récidive dans $30 \%$ des cas.

Les indications et l'étendue du curage sont également controversées. La lésion primitive de la verge est souvent surinfectée, comme dans la majorité des cas de notre série. Pour Mukamel (18), 40 à $60 \%$ des patients présentent des adénopathies. Dans $50 \%$ des cas, il s'agit d'une atteinte métastastique et dans les autres cas d'une simple réaction inflammatoire. L'apparition ou la persistance d'adénopathies après traitement de lésion primitive et antibiotiques + antiinflammatoires est fortement suspecte d'envahissement métastatique $(1,18)$. Cependant dans 5 à $20 \%$ des cas, l'absence de ganglions cliniquement accessibles n'exclue pas l'existence de micro-métastases. Pour Mukamel (18) la précocité de l'envahissement ganglionnaire semble en rapport avec le degré de différenciation cellulaire, la taille de la tumeur, l'extension aux corps caverneux, la présence d'embols lymphatiques ou vasculaires. Pour Hardner et coll. (14), lorsque les corps caverneux ne sont pas envahis, seuls $5 \%$ des patients présenteraient un envahissement ganglionnaire contre $65 \%$ lorsquils le sont.

Selon Cabanas (4), les premiers relais métastatiques s'effectuent dans les ganglions de la crosse de la saphène. Pour Adjiman (1), le curage lymphatique iliaque bilatéral, en l'absence de ganglion palpable, s'accompagne d'une morbidité élevée (50\% des cas). C'est pourquoi la biopsie exérèse d'un groupe ganglionnaire proche de la saphène (groupe 'sentinelle) est proposée par certains auteurs $(4,25,26)$ pour discriminer les malades $\mathrm{N}+$. Pour Cabanas et coll. (4), quand ce groupe ganglionnaire n'est pas envahi, les autres groupes ganglionnaires sont indemnes, dans $90 \%$ des cas. Cependant, de nombreux auteurs tels que Wespes $(30)$ et Dekernion (5) ont rapporté des développements secondaires de métastases inguinales ou iliopelviennes chez des patients avec biopsie bilatérale négative des groupes ganglionnaires sentinelles.

Il existe par ailleurs une relation directe entre l'envahissement ganglionnaire et la durée de survie. Dans la série de 199 patients de Srinivas (25), la médiane de survie à 5 ans est de $56 \%$ lorsqu'un ganglion unilatéral est envahi et de seulement $9 \%$ lorsque la dissémination est bilatérale. Après analyse de leur série de 65 patients Dougal et coll. sont partisans d'une lymphadénectomie systématique. Leur taux de survie à 5 ans est de $88 \%$ pour le stade II, $66 \%$ pour le stade III. Il chute à $38 \%$ au stade II et à 0 $\%$ au stade III lorsque la lymphadénectomie n'a pas été effectuée.

Il nous semble donc que, sauf pour les petites tumeurs vues précocement qui peuvent bénéficier de thérapeutiques conservatrices, seute l'amputation qui assure la guérison locale, et le curage ganglionnaire, malgré sa morbidité, assurent un traitement efficace des cancers épidermoïdes du pénis. Cette attitude fut déjà recommandée en 1984 par Douglas (7) qui préconise le curage ganglionnaire systématique et précoce. Cependant, comme le signale cet auteur après une expérience de 101 lymphadénectomies (6), la morbidité est élevée puisquili signale $50 \%$ de nécroses cutanées, $16 \%$ de lymphorrées, $14 \%$ d'infections cutanées, $9 \%$ de lymphoedèmes, et 6 $\%$ de thrombophlébites.

\section{Aspects physiopathologiques :}

Les travaux épidémiologiques de Riveros (23) montrent une répartition très particulière de ce cancer avec deux zones de haute fréquence, équatoriale et tropicale, toutes 2 chaudes et humides : Amérique du Sus (Brésil, Paraguay) et Asie (Java, Chine, indochine). Les pays d'Afrique où l'on pratique la circoncision, observent très rarement cette pathologie. Pour Riveros (23), suite à son analyse épidémiologique au Paraguay, l'absence de circoncision, le manque d'hygiène et la promiscuité sexuelle favorisent l'apparition de ce cancer chez l'homme et contribueraient à l'apparition de cancer du col de l'utérus chez la femme. Crahan (13) note une corrélation entre la fréquence des cancers du col de l'utérus chez les femmes noires de milieux sociaux défavorisés et la fréquence des cancers du pénis chez leurs partenaires.
En 1986, Villa et coll. (29) ont extrait des tumeurs du DNA de papilloma virus chez 8 patients sur 18 porteurs de tumeur du pénis (44 $\%$ ). 7 de ces DNA correspondaient au HPV 18 et 1 au HPV 11. Déjà en 1956, Pratt signalait le rôle carcinogène du smegma de l'homme. Par ailleurs, le rôle de certains papilloma virus est bien connu dans la génèse des cancers du col chez la femme (3). Il existe une relation entre ces papilloma virus et les tumeurs du pénis et du col de l'utérus. Une étude japonaise récente vient de confirmer le caractère transformant direct des virus papilloma virus associé aux lésions malignes. Tsunokawa et son équipe (28) ont démontré que l'ADN extrait d'une tumeur du col utérin contient tout ou partie du génome du HPV 16 et transforme en culture les fibroblastes de souris. La circoncision, en favorisant une meilleure hygiène dans les pays en voie de développement, limiterait une macération chaude et humide favorable à l'action de ces virus ou d'un cofacteur tumoral.

Il nous apparait donc de plus en plus indispensable de rechercher en péniscopie après application d'acide acétique à $5 \%$ les localisations papillomateuses du gland et de les traiter. En particulier, celles des souches impliqués dans les néoplasies intraépithéliales (HPV 16, 18, 33). Cependant leur identification virologique et immunologique n'est pas encore de pratique courante. Les HPV 6 et 11 ne sont pas impliqués dans l'apparition de néoplasies intraépithéliales. Ils sont responsables de l'apparition de condylomes plans ou de condylomes exophytiques sans atypie cellulaire. Comme le signale Monsonego (17), 40 à $50 \%$ des partenaires de femmes présentant des condylomes ou des néoplasies intra-épithéliales ont des lésions virales infra-cliniques. Chez ces porteurs, on identifie des partenaires à haut risque carcinogène (HPV 16) et des partenaires à bas risque (HPV 6 et 11). L'homme doit donc être considéré comme à bas rique de transformation cancéreuse intrinsèque mais avant tout comme co-facteur à l'origine de recontamination, d'infestation et de transmission de la maladic. Seul le dépistage par péniscopie et colposcopie, ainsi que le traitement local de ces lésions pourrait limiter le risque de dissémination, ou l'apparition d'une néoplasie intraépithéliale chez la femme, ou plus rarement l'apparition d'un cancer du pénis chez l'homme.

\section{CONCLUSION}

Les cancers du pénis sont rares. La curiethérapie ou le laser yag permettent un traitement conservateur dans certains cas vus précocement. Sinon leur traitement repose toujours sur l'amputation qui seule peut assurer une guérison locale.

La morbidité élevée du curage ganglionnaire 
explique que l'on puisse se contenter soit d'une surveillance clinique étroite dans les stades précoces, soit de la biopsie du groupe sentinelle. En cas d'envahissement ganglionnaire, éventualité fréquente car les patients consultent souvent tardivement, l'amputation associée au curage ganglionnaire demeure le seul traitement efficace. Le pronostic demeure cependant encore très sombre.

La découverte de papilloma virus oncogène dans ces tumeurs incite à dépister par péniscopie les patients porteurs et à les traiter ainsi que leurs partenaires.

\section{REFERENCES}

1 - Adjiman S, Zerbib M, Flam T, Desligneres S, Debré B, Steg A. Traitement du cancer épidermoïde de la verge. Presse Méd, 1989. 32:1117-1119.

2- Bandieramonte G, Lepera P, Marchesini R, Andreola S, Pizzocaro G - Laser microsurgery for superficial lesions of the penis. J. Urol, 1987. 138:315-319.

3 - Baudeman S, Kremsdorf D, Croissant O, Jablonsha S, Wain-Hobson S, Orth G. A novel type of human papillomavirus associated with genital neoplasias Nature, 1986, 321:246-249.

4 - Cabanas R.M. An approach for the treatment of penile carcinoma. Cancer, 1977, 39:456-461.

5 - Dekernion J.B., Tynberg P, Pershy L, Fegen J.P. Carcinoma of the penis. Cancer, 1973, 32 : 1256-1262.

6 - Douglas E.J., Richard K.L. Complications of groin dissection in penile cancer. Urology, 1984, 24 : 312-314.

7 - Douglas E.J., Richard K.L. Management of regional lymph nodes in penile carcinoma. Urology, 1984, 24:308-311.

8 - Dougal W.S., Kirchner P.K., Edwards R.H. Killion L.T. Treatment of carcinoma of the penis: the case for primary lymphadenectomy. J, Urol. 1986, 136:38-41.

9- Duncan W, Jackson S.M. The treament of early cancer of the penis with megavoltage $x$ rays. Clin Radiol, 1972, 23:246-248.
10 -El Demiry M, Oliver D, Hopestone H, Blandy J.P. Reappraisal of the role of radiotherapy and surgery in the management of carcinoma of the penis. Brit. J. Urol, 1984, 56:724-728.

11 -Fraley E, Zhang G, Manivel C, Niehans G. The role of ilioinguinal lymphadenectomy and significance of histological differentiation in treatment of carcinoma of the penis. J. Urol, 1989, 142:1478-1482.

12 -Fraley E, Zhang G, Sazama R, Lange PH. Cancer of the penis : prognosis and treament plans. Cancer, 1985, 55:1618-1624.

13-Graham S, Priore R, Graham M, Browne R, Burnett $W$, West $D$. Genital cancer in wives of penile cancer patients Cancer, 1979, 44:1870-1874.

14 -Hardner G, Hanaiaph TB, Murphy GP, Albert DJ, Movre RH. Carcinoma of the penis : analysis of therapy in 100 consecutives cases. J. Urol 1972 , $108: 428-435$.

15 -Jackson E, Fowler J. Sentinel lymp mode biopy for staging penile cancer. Urology, 1984, 23 : 352-354.

16 -Kaye V, Zhang G, Dehner L, Fraley E. Carcinoma in situ of penis. Urology, 1990, 36:479-481.

17 -Monsonego J. Dysplasie du col utérin et papillomavirus humains Maloine, Paris, 1988.

18 - Mukamel E, Dekernion J.B. Early versus delayed lymphonode, dissection versus no lymph node, dissection in carcinoma of the penis. Urol. Cin, North AM., 1987, 14 : 707-711.

19 -Neison R.P., Derrick F.C. Allen W.R. Epidermoid carcinoma of the penis. Br. J. Urol, 1982, 54 : 172-175.

20-Perinetti E, Crane D.B., Catalona W.J., Unreliability of sentinel lymph node biopsy for staging penile carcinoma. J. Urol, 1980, 124 : 734-735.

21 -Pierquin B, Bunescu U, Chassagne D, Jomain J. La radiotherapie des cancers de la verge par l'iridium 192. J. Urol, Nephrol. 1970, 76:1-8.

22 - Pratt T, Heins H.R., Latham E, Dennis E.J., Mac Iver P.A. Carcinogenic effect of human smegma : experimental study Cancer, 1956, 9:671-680.

23 -Riveros M, Lebron R. Geographical pathology of cancer of the penis. Cancer, 1963, 16:798-811.
24 -Srinivas V, Alikhan S. Treatment of cancer of the penis : a review. Int. Urol. Nephrol., 1989, 21 : 185-193.

25-Srinivas V, Morse M.J., Herr H.W., Sogani P.C., Whitmore J.R. Penile cancer : relation of extent of nodal metastasis to survival. J Urol, 1987, 137 : 880-881.

26 -Staehler G, Chaussy C, Jocham D, Schmiedt E. The use of neodymium yag lasers in urology : indications, technique and critical assessment J. Urol, 1985, 134:1155-1160.

27 -Suchaud J.P., Kantor G, Richaud P, Mage P, Lamarche P, Dilhuydy J.M., Pigneux J. Curietherapie des cancers de la verge : Analyse d'une série de 53 cas J. Urol. 1985, 95:27-31.

28 -Tsunokawa Y, Takebe N, Tatshuiro K, Terada M, Siguimura J. Transforming activity of human papillomavirus type 16 DNA sequences in cervical cancer. Proc. Nat. Acad. Sci. USA, 1986, 83 : 2200-2203.

29 - Villa LL, Lopes A. Human papillomavirus DNA sequences in penile carcinoma in Brazil. Int. J. Cancer, 1986, 37:853-855.

30-Wespes E, Simon J, Schulman C.C. Cabanas approach : is sentinel node biopy reliable for staging penile carcinoma? Urology, 1986, 28 : 278-279.

RESUME : Le cancer épidermoïde du pénis est peu fréquent. Au stade précoce, la curietherapie et le laser permettent un traitement conservateur. Au stade avancé, le traitement de la lésion primitive repose sur l'amputation. L'existence d'un envahissement ganglionnaire conditionne le pronostic. La conduite à tenir à l'égard des ganglions reste encore controversée. Le curage ganglionnaire reste le seul traitement efficace des ganglions métastatiques mais sa morbidité est élevée. La découverte de génomes de papilloma virus dans de telles tumeurs incite à dépister en péniscopie ces lésions virales et à les traiter. Mots clés : Cancer épidermoïde - pénis - ganglions traitement. Andrologie 1991, 1 : 71-73. 